\title{
Characteristics of Clinical and Imaging Findings in Adolescent Lumbar Spondylolysis Associated with Sports Activities
}

\author{
Akira Hirano $^{1}$, Tsuneo Takebayashi ${ }^{*}$, Mitsunori Yoshimoto', Kazunori Ida ${ }^{1}$, Tosihiko Yamashita ${ }^{1}$ and Kazuhiko Nakano ${ }^{2}$ \\ ${ }^{1}$ Department of Orthopaedic Surgery, Sapporo Medical University School of Medicine, Japan \\ ${ }^{2}$ Department of Orthopaedic Surgery, Nishioka Daiichi Hospital, Japan
}

\begin{abstract}
Background: Adolescent lumbar spondylolysis is considered to be a fatigue fracture caused by repeated mechanical stress and sports activities are often thought to be the cause. The incidence of lumbar spondylolysis has been reported to be generally $3 \%-6 \%$, and 4\%-10\% among patients complaining LBP. In athletes, the incidence of lumbar spondylolysis during adolescence has ranged from approximately $15 \%$ to $50 \%$. The purpose of this study was to prospectively investigate to determine the imaging and pain characteristics of spondylolysis on lumbar extension among adolescent patients with Low Back Pain (LBP) who played sports.
\end{abstract}

Methods: 100 patients (a mean age of 14.8 years) were selected as the subjects and were investigated sports activities, duration of LBP, and pain with lumbar repetitive extension. Plain X-ray, CT, and high-intensity signal changes in the pedicle on T2 MRI were examined for diagnostic indications of lumbar spondylolysis.

Results: Spondylolysis was confirmed by plain X-ray in 34 patients, 8 were confirmed on CT. Spondylolysis was eventually identified in 42 of the 100 patients (42.0\%). High-intensity signal changes in the pedicle on T2 MRI were recognized in 29 cases. Pain with lumbar extension occurred in 69 of the 100 patients $(69.0 \%)$ including 34 of the 42 patients with spondylolysis (81.0\%) and 35 of the 58 patients without spondylolysis $(60.3 \%)$. Therefore, the sensitivity of the lumbar extension was $81.0 \%(34 / 42)$, and its specificity was $39.7 \%(23 / 58)$

Conclusion: Among adolescent athletes, the incidence of lumbar spondylolysis was $42.0 \%$, and of the 69 patients with pain with lumbar extension, $34(81.0 \%)$ were confirmed to have spondylolysis. Pain with lumbar extension appears to be a good screening indicator for adolescent lumbar spondylolysis.

Keywords: Adolescent lumbar spondylolysis; Athletes; MRI

\section{Introduction}

Adolescent lumbar spondylolysis is considered to be a fatigue fracture caused by repeated stress between the zygapophyseal joint and the adjacent areas, and sports activities are often thought to be the cause. While it has been reported that early diagnosis and therapy increase the rate of union, the incidence of pseudarthrosis remains high in clinical settings.

The incidence of lumbar spondylolysis has been reported to be between $3 \%-6 \%[1-3]$ and $4 \%-10 \%$ among patients with Low Back Pain (LBP) as the chief complaint [4,5]. In athletes, the incidence of lumbar spondylolysis during adolescence ranges from approximately $15 \%$ to $50 \%[6,7]$. Hori et al. [7] studied patients (10-18 years of age) who played sports almost daily and had pain with lumbar extension, and they reported that the incidence of lumbar spondylolysis in adolescent athletes was as high as $50.6 \%(45 / 89)$ [7]. In the present study, the clinical symptoms and imaging findings, including those from plain X-ray, Computed Tomography (CT), and Magnetic Resonance Imaging (MRI), of adolescent athletes presenting with LBP were prospectively investigated to determine the incidence of lumbar spondylolysis and the usefulness of pain with lumbar repetitive extension as a screening indicator for diagnosis of spondylolysis.

\section{Methods}

Written informed consents were obtained from all the patients who participated in this study, and the protocols were approved by our ethics committee.

A prospective study was conducted in patients 8-18 years of age, who played sports almost daily and visited the hospital with LBP as the chief complaint from January to October, 2008. One hundred patients (76 men and 24 women) with a mean age of 14.8 years were selected as the subjects and included 31 baseball players, 24 soccer players, 9 volleyball players, 9 basketball players, and 27 players involved in other sports (table tennis, swimming, etc.). The duration of LBP was $<2$ weeks in $51,2-4$ weeks in $5,1-3$ months in 23 , and $\geq 3$ months in 21 patients. At the time of the initial visit to the hospital, the patients' clinical symptoms, such as LBP and leg pain, were investigated. Generally, LBP with lumbar extension is regarded as the characteristic symptom of lumbar spondylolysis. Pain on only initial lumbar extension may be a false-positive in predicting the presence of spondylolysis. Therefore, the usefulness of LBP with lumbar repetitive extension as a screening indicator for lumbar spondylolysis in adolescent athletes was evaluated within physiological range, not on hyperextension. We performed plain X-ray, CT, and T2-weighted (T2) MRI in all patients. A GE Signa HDx 1.5T scanner with a spine coil (GE Healthcare, Milwaukee, WI, USA) was used to perform T2 MRI. X-ray and CT findings and highintensity signal changes in the pedicle on T2 MRI were used by one of the co-authors, who was not involved in the examination of the patients, to clinically diagnose lumbar spondylolysis.

\section{Results}

Spondylolysis was confirmed by plain X-ray in 34 patients. Of the 66 patients in whom spondylolysis could not be confirmed by plain $\mathrm{X}$-ray, 8 were confirmed to have spondylolysis on CT. Spondylolysis was eventually identified in 42 of the 100 patients (42.0\%). Highintensity signal changes in the pedicle on T2 MRI were recognized in 29 cases: 9 of the 34 patients (26.5\%) who were confirmed to have

*Corresponding author: Tsuneo Takebayashi, Department of Orthopedic Surgery School of Medicine, Sapporo Medical University, Hokkaido 060-8543, Japan, Tel: 81-11-611-2111; Fax: 81-11-641-6026; E-mail: takebaya@sapmed.ac.jp

Received July 08, 2012; Accepted September 14, 2012; Published Septembe 19, 2012

Citation: Hirano A, Takebayashi T, Yoshimoto M, Ida K, Yamashita T, et al (2012) Characteristics of Clinical and Imaging Findings in Adolescent Lumba Spondylolysis Associated with Sports Activities. J Spine 1:124. doi:10.4172/21657939.1000124

Copyright: $\odot 2012$ Hirano A, et al. This is an open-access article distributed under the terms of the Creative Commons Attribution License, which permits unrestricted use, distribution, and reproduction in any medium, provided the original author and source are credited. 
spondylolysis on plain X-ray, 8 of the 8 patients (100\%) who were found to have spondylolysis on CT, and 12 of the 58 patients $(20.7 \%)$ who had no spondylolysis (Table 1).

Overall, 69 of the 100 patients $(69.0 \%)$ had LBP with lumbar extension, including 34 of the 42 patients with spondylolysis $(81.0 \%)$ and 35 of the 58 patients without spondylolysis (60.3\%) although there were some differences of the degree of extension among the patients. Therefore, the sensitivity of LBP with lumbar extension was $81.0 \%$ (34/42), and its specificity was 39.7\% (23/58) (Table 2). The percentage of patients who do not have pain with lumbar extension and spondylolysis was $74.2 \%$.

Five patients also complained of leg pain (5.0\%) and 2 of them were confirmed to have lumbar disc herniation on MRI.

\section{Discussion}

According to a 2010 study by Sakai et al., the incidence of lumbar spondylolysis in Japan was $5.9 \%$ [8]. They found that the incidence was much higher (30\%) in professional Japanese soccer and baseball players. Furthermore, they reported that the incidence of lumbar spondylolysis in professional athletes was more than 5 times the incidence in the general Japanese population and varied depending on ethnicity, sex, family history, relevant disease, and sports activity. Previously, we reported that $50.6 \%$ (45/89) of athletes presented with LBP with lumbar extension [7]. Kujala et al. [9] documented lumbar pain in $45 \%$ of athletes during the growing stage but only in $18 \%$ of developing children who did not play sports [9]. Micheli and Wood examined patients who visited a sports clinic with LBP as the chief complaint and reported the incidence of spondylolysis to be $5 \%$ in adults, but $47 \%$ in adolescents [5]. According to a study by Rossi et al., the incidence of lumbar spondylolysis among the general public, not limited to adolescents, was $4 \%-7 \%$, but was much higher among athletes (13.5\%) [6]. In the present prospective study, the subjects included patients 8-18 years of age, who presented with LBP and played sports almost daily, irrespective of the presence/absence of LBP with lumbar extension. The results demonstrated that the incidence of spondylolysis was high, $42.0 \%$ (42/100). Thus, it is important to note that lumbar spondylolysis has a high incidence in adolescent athletes.

The relationship between spondylolysis and LBP with lumbar extension is demonstrated in table 2 . Of the 42 spondylolysis patients, 34 had LBP with lumbar extension, and the sensitivity for LBP with lumbar extension was $81.0 \%$. Of the 58 patients without LBP with lumbar extension, 23 did not have spondylolysis, giving a specificity of $39.7 \%$. Thus, LBP with lumbar extension is a useful indicator when screening for spondylolysis. If LBP with lumbar extension is present, MRI and CT should be performed to avoid a misdiagnosis. The

\begin{tabular}{|l|l|}
\hline Spondylolysis & MRI high-intensity \\
\hline Spondylolysis (+) by plain X-ray : 34 pts & 9 pts \\
\hline $\begin{array}{l}\text { Spondylolysis (-) by plain X-ray (66 patients) } \\
\text { and spondylolysis (+) by CT : } 8 \text { pts }\end{array}$ & $8 \mathrm{pts}$ \\
\hline Spondylolysis (-) by plain X-ray and CT : 58 pts & 12 pts \\
\hline
\end{tabular}

Table 1: Relationship between Spondylolysis (plain X-ray $\cdot C T$ ) and MRI highintensity signal changes.

\begin{tabular}{|l|l|l|}
\hline & Spondylolysis (+) & Spondylolysis (-) \\
\hline LBP with lumbar extension (+) & 34 pts & 35 pts \\
\hline LBP with lumbar extension (-) & 8 pts & 23 pts \\
\hline
\end{tabular}

LBP with lumbar extension (+) : $69 / 100$ patients

Sensitivity : $81.0 \%$ Specificity of $: 39.7 \%$

Table 2: Relationship between LBP with lumbar extension and spondylolysis. percentage of patients who do not have pain with lumbar extension and spondylolysis was $74.2 \%$ i.e., in the absence of LBP with lumbar extension, the probability of spondylolysis is low, and it is not necessary to promptly perform additional imaging tests, such as CT or MRI, although careful follow-up with periodic observation is required.

Fifty-eight patients did not have spondylolysis at the time of the initial visit, and of these patients, 12 had high-intensity signal changes in the pedicle on T2 MRI. Sairyo et al. [10] referred to this condition as early-stage spondylolysis [10]. In other words, although spondylolysis cannot be seen on plain X-ray or CT, changes caused by mechanical stress to the pedicle area just before separation can be seen on MRI, thus indirectly confirming spondylolysis. Therefore, when high-intensity signal changes in the pedicle are recognized, careful monitoring is necessary even if spondylolysis cannot be confirmed. Thus, it appears valid to instruct patients to temporarily discontinue sports activities.

Many points remain unknown about the natural course in patients with high-intensity signal changes in the pedicle on T2 MRI. The data in table 1 demonstrates that, of the 8 patients with confirmed spondylolysis on CT but not on plain X-ray, all had high-intensity signal changes in the pedicle on T2 MRI, and this was considered to be indicative of comparatively new spondylolysis. However, it was not confirmed whether the 12 patients (early-stage spondylolysis) with no spondylolysis on X-ray and CT but with high-intensity changes in the pedicle on T2 MRI would eventually develop spondylolysis. It is believed that even if high-intensity signal changes caused by mechanical stress to the pedicle are observed, spondylolysis may not necessarily develop. However, it is possible that the incidence of this occurring is very high among athletes whose lumbar spines are constantly subjected to mechanical stress. MRI would have to be performed to confirm this point in many adolescent athletes with or without LBP, but such studies would be impractical.

This was a prospective study on the clinical and imaging findings of adolescent athletes (8-18 years), presenting with LBP to determine the incidence of lumbar spondylolysis. Among these athletes, the incidence of lumbar spondylolysis was $42.0 \%(42 / 100)$. Overall, 69 of the 100 patients $(69.0 \%)$ had LBP with lumbar extension, including 34 of the 42 patients with spondylolysis (81.0\%). A limitation of this study is that examination and investigations were all performed at one time, therefore it is required that a consecutive investigation of clinical symptoms and imaging findings, especially in patients with negative $\mathrm{X}$-rays and CT scan but with MRI high-intensity signal changes in the pedicle.

\section{References}

1. Wiltse LL (1962) The etiology of spondylolisthesis. J Bone Joint Surg Am 44A: 539-560.

2. Roche MB, Rowe GG (1951) The incidence of separate neural arch and coincident bone variations; a survey of 4,200 skeletons. Anat Rec 109: 233 252.

3. Fredrickson BE, Baker D, McHolick WJ, Yuan HA, Lubicky JP (1984) The natural history of spondylolysis and spondylolisthesis. J Bone Joint Surg Am 66: 699-707.

4. Rossi F, Dragoni S (1994) Lumbar spondylolysis and sports. The radiologica findings and statistical considerations. Radiol Med 87: 397-400.

5. Micheli LJ, Wood R (1995) Back pain in young athletes. Significant differences from adults in causes and patterns. Arch Pediatr Adolesc Med 149: 15-18.

6. Rossi F (1978) Spondylolysis, spondylolisthesis and sports. J Sports Med Phys Fitness 18: 317-340.

7. Hori K, Takebayashi T, Yamashita T, Nakano K (2006) Spondylolysis on the athletes of the growth period. MB Ortho 19: 9-14. 
Citation: Hirano A, Takebayashi T, Yoshimoto M, Ida K, Yamashita T, et al. (2012) Characteristics of Clinical and Imaging Findings in Adolescent Lumbar Spondylolysis Associated with Sports Activities. J Spine 1:124. doi:10.4172/2165-7939.1000124

8. Sakai T, Sairyo K, Suzue N, Kosaka H, Yasui N (2010) Incidence and etiology of lumbar spondylolysis: review of the literature. J Orthop Sci 15: 281-288.

9. Kujala UM, Taimela S, Erkintalo M, Salminen JJ, Kaprio J (1996) Low-back pain in adolescent athletes. Med Sci Sports Exerc 28: 165-170.
10. Sairyo K, Katoh S, Takata Y, Terai T, Yasui N, et al. (2006) MRI signal changes of the pedicle as an indicator for early diagnosis of spondylolysis in children and adolescents: a clinical and biomechanical study. Spine (Phila Pa 1976) 31: $206-211$ 